\title{
Municipalización Educativa: Reflexiones en Base a Tres Experiencias en Latinoamérica
}

\author{
Educational Municipalization: Reflections Based on Three Experiences in Latin \\ America
}

\author{
Gisella Azucena PÉrez lozada \\ gisella.perez1@unmsm.edu.pe \\ https://orcid.org/0000-0002-4534-200X \\ Universidad Nacional Mayor de San Marcos
}

\begin{abstract}
RESUMEN:
El objetivo de este artículo es revisar el proceso de municipalización de la gestión educativa que se gestó durante el periodo 2007 al 2011 en Perú y reflexionar sobre otros modelos en nuestra región. Por lo tanto, revisaremos los modelos peruano, brasilero y chileno considerando que no existe un modelo único. A partir de un enfoque cualitativo que considera una revisión sistemática hemos analizado documentación que incluyó criterios de origen, temática y temporalidad. Los resultados indican que en el caso peruano hubo un apresuramiento en la implementación de la municipalización y como consecuencia hubo duplicidad de funciones; mientras que, Chile ha empezado a desmunicipalizar la gestión educativa, debido a que el enfoque mercantilista socavaba la educación pública. En el caso de Brasil se presenta una educación pública que tiene en todos sus niveles de gobierno una autonomía total, lo que facilita la municipalización de la educación pública sin que eso signifique que no existan complicaciones en la práctica. Podemos concluir que, en nuestra región, la municipalización ha tenido múltiples enfoques, orientaciones y el tema de descentralizar la educación tienen muchas aristas por explorar.
\end{abstract}

\section{ABSTRACT:}

The objective of this article is to review the process of municipalization of educational management that took place during the period 2007 to 2011 in Peru and to reflect on other models in our region. Therefore, we will review the Peruvian, Brazilian and Chilean models considering that there is no single model. Using a qualitative approach that considers a systematic review, we have analyzed documentation that included criteria of origin, subject matter and temporality. The results indicate that in the Peruvian case there was a rush in the implementation of municipalization and as a consequence there was a duplication of functions; while Chile has begun to de-municipalize educational management, due to the mercantilist approach undermining public education. In the case of Brazil, a public education is presented that has total autonomy at all levels of government, which facilitates the municipalization of public education without this meaning that there are no complications in practice. We can conclude that, in our region, municipalization has had multiple approaches, orientations, and the issue of decentralizing education has many edges to explore.

\section{Palabras Clave:}

Municipalización; descentralización; gestión educativa; calidad educativa.

\section{KEYWORDS:}

Municipalization; decentralization; educational management; educational quality. 


\section{Introducción}

La gestión en el campo educativo de diversos países del mundo como Finlandia, Estados Unidos, el Reino Unido y Canadá entre otros, nos ilustra cómo se manifiestan los sistemas educativos a nivel de gobiernos locales y cómo podrían tener un efecto directo en la eficiencia y calidad educativa. Esto último comulga con la cuarta meta de la Agenda 2030 para el Desarrollo Sostenible, es decir, asegurar una educación de calidad. Según esta agenda, los países miembros de la Organización de las Naciones Unidas para la Educación, la Ciencia y la Cultura (Unesco, 2016) se comprometen a desarrollar políticas públicas que promuevan sistemas educativos que garanticen la prestación de servicios de forma eficaz y promuevan la participación de los docentes, familias y comunidad en general.

En la actualidad, menos de un tercio de países miembros de la Organización para la Cooperación y el Desarrollo Económico (OCDE, 2018) tienen sistemas educativos en donde la mayoría de decisiones son aplicadas a nivel del gobierno central y aproximadamente, un cincuenta por ciento son a nivel de gobierno local o a nivel de institución educativa. Estudios realizados por esta misma institución indican que la descentralización educativa parte de la premisa que los gobiernos locales y las escuelas autónomas están mejor ubicados para conocer las urgencias de la localidad y sus estudiantes.

La descentralización de la gestión educativa a nivel local como una alternativa en nuestra región sigue vigente, lo que demuestra que es posible gestionar la educación desde gobiernos locales, donde Brasil, Argentina y Colombia son ejemplos de ello, con contextos y características propias (Rivas, 2015). Un análisis de nuestra experiencia comparada con la de otros países de la región sería ideal para encontrar una nueva versión a aplicarse en un futuro; además, de revisar la influencia de esta forma de delegación de funciones en la gestión educativa.

Uno de los desafíos del Estado peruano ha sido promover una educación de calidad con el objetivo de lograr el progreso de sus ciudadanos a través de políticas nacionales que promuevan la descentralización de la gestión de la educación. No obstante, la trama burocrática, los lineamientos indefinidos y duplicidad de responsabilidades pueden transformarse en barreras que obstaculicen que se proporcione un servicio óptimo a la comunidad (Unesco, 2020).

En el 2007 se propuso trasladar la gestión de la educación a las instancias locales por medio del Plan Piloto de Municipalización de la Gestión Educativa (Ppmge). Una decisión política que involucró un proceso largo y complejo del cual debemos extraer lecciones y reevaluar la posibilidad de replantearlo una vez más. Entonces, es pertinente reflexionar sobre qué es municipalizar la gestión educativa, qué comprende y por qué el plan piloto se dio por finalizado en el año 2011.

La literatura revisada define la descentralización como la orientación de políticas públicas, es decir, es una decisión política, que se opta cuando un sistema centralizado no alcanza los niveles de eficiencia deseados y como toda opción política posee ventajas y desventajas. Entre las ventajas se puede considerar democratizar decisiones que impacten directamente a una comunidad al otorgarle voz al ciudadano; así como puede convertirse en una herramienta para una gestión eficaz y determinar diferentes niveles de responsabilidad (Instituto Internacional de Planificación Educativa [IIPE]-Unesco, 2018).

Para IIEP-Unesco (2021) existen dos formas principales de descentralización: la funcional y la territorial. Con relación a la descentralización funcional esta se refiere a la distribución de poderes que operan en paralelo; mientras que, la descentralización territorial está relacionada a la delegación de poderes a través de gobiernos subnacionales. A su vez este tipo de descentralización se divide en desconcentración, cuando se establecen oficinas regionales de un sector gubernamental, pero este retiene el control; la delegación cuando solo se delegan tareas, pero no autoridad y finalmente, la devolución cuando si se delega autoridad a gobiernos locales volviéndose independientes y autónomos. 
Para poder determinar si estamos encaminados hacia la mejora de la calidad educativa es necesario entonces determinar qué es este término y cómo se puede medir. Según la Unesco (2016) el término calidad educativa puede definirse bajo dos enfoques, el enfoque conductista que se concentra en la observación de herramientas de medición y el constructivista que observa la construcción de aprendizajes para el desarrollo completo de los estudiantes y cómo dan utilidad a lo que aprenden. Además, esta organización propone cinco categorías dentro de la calidad educativa: equidad, relevancia, pertinencia, eficacia y eficiencia. Creemos pertinente ahondar en estas dimensiones y preguntar la relevancia, la misma que se relaciona con las finalidades y funciones.

Precisamos que la pertinencia resalta que el estudiante se encuentra en medio del proceso educativo y por lo tanto, toda práctica educativa debe adaptarse a su entorno. Asimismo, la equidad busca garantizar que la educación proporcione oportunidades para todos; mientras que la eficacia mide los logros de las metas establecidas las cuales deben ser claras aunada a que mide la gestión y el uso apropiado de los recursos educativos.

Según el Banco Interamericano de Desarrollo (BID, 2019), la gestión del sistema educativo debe reflejar una visión global del mismo, que planifique de forma integral y eficiente los procesos de gestión y asignación de recursos, identificando qué necesitan aquellos encargados de la toma de decisiones en todos los estratos de gestión, desde el nivel central hasta el ámbito de los docentes. Además, se identifica que existen seis herramientas principales de gestión: infraestructura física y acompañamiento, instituciones educativas, recursos humanos y financieros, estudiantes y aprendizaje y finalmente, contenidos digitales para aprendizajes y formación docente.

$\mathrm{Al}$ grupo de herramientas anteriormente mencionado se agregan las condiciones estructurales dentro de las cuales se incluye la infraestructura tecnológica y la gobernanza e institucionalidad. La infraestructura tecnológica se transforma en una importante herramienta para llevar a cabo la gestión estratégica debido a que una gestión propiamente automatizada permite medir la eficiencia de la gestión educativa a través de indicadores claves para los distintos niveles del sistema educativo, los indicadores de aprendizaje (BID, 2019).

El Banco Mundial (2018) señala que los sistemas educativos son un conjunto de instituciones, acciones, procesos y la interacción de actores, como profesores, padres de familia, políticos, la comunidad, entre otros; los cuales debaten temas referentes a desarrollo curricular, gestión educativa y gobernabilidad. Los sistemas educativos tienen como objetivo central el aprendizaje, aunque muchas veces se observa que no existe coherencia entre un sistema educativo y el aprendizaje, todo esto debido a complejidades que van desde la financiación estatal, burocracia y factores técnicos y políticos. Una gestión deficiente no garantiza un apropiado servicio y por ende afecta la calidad educativa.

Canazas y Márquez (2015) indican que municipalizar la gestión educativa no es algo nuevo y que ha tenido presencia desde los inicios de la República. El planteamiento reciente busca plantear un nuevo mecanismo de desarrollo educativo, descentralizando la toma de decisiones, dándole voz a la población, y adaptando políticas nacionales al entorno propio de cada región, localidad y/o caserío. Para Moreno (2021) la descentralización educativa tiene como objetivo esencial el desarrollo humano lo cual se puede lograr a través de un proceso de planificación de políticas públicas que incluya a todos los integrantes de la comunidad. El Ministerio de Educación de Perú (Minedu, 2015) presenta los lineamientos para gestionar la educación pública de manera descentralizada constituyéndose en un marco de referencia que establece los objetivos, roles, y funciones del sistema educativo.

Kameshwara et al. (2020) hacen un análisis de la relación entre descentralización y calidad educativa basado en estudios realizados sobre la prueba PISA (Programa de Evaluación Internacional de Estudiantes). El tema de descentralización y sus efectos en la gestión de la educación es un aspecto recurrente que posee muchas aristas por explorar y determinar, si las expectativas y beneficios planteados por gobiernos nacionales se cumplen bajo esta política de Estado. Ríos (2020) nos invita a reflexionar sobre los entes intermediarios entre el órgano rector y los centros educativos en el Perú llamados Unidades de Gestión Educativa Local (UGEL), los cuales tienen cerca de 20 años de creación. Por un momento podríamos detenernos a considerar 
si estos organismos de gestión demuestran que la descentralización se concretiza a través de ellos, pero los estudios indican que las labores que se llevan a cabo son de carácter administrativo, operacional, no político y no democrático.

Para Ríos (2017) lo referente a municipalizar es una oportunidad para la administración del sistema educativo conducente a optimizar el servicio que brindan a la comunidad y al mismo tiempo permite que todos los actores del sistema educativo interactúen de forma eficiente. Existen muchos modelos de descentralización de gestión educativa que se hace necesario revisar, como el caso chileno que es un modelo atípico e inapropiado con un desempeño negativo (Cerro, 2020). El tema de la descentralización educativa a través de diferentes modelos nos invita a revisar antecedentes de los componentes de estas políticas públicas. Otro aporte a este tema en particular nos lo dan Bellei et al. (2018) al ilustrar el proceso de desmunicipalización por el que está pasando esta nación.

Jiménez (2019) sostiene que gestionar en el sector educativo involucra construir una relación entre profesores, personal directivo, alumnado y la sociedad en general, así como interactuar para lograr metas de mejora de la calidad de la educación y fortalecer la institucionalidad. Adicionalmente, Chacón (2014) indica que gestionar en el campo educativo es aplicar saberes y prácticas organizacionales que van más allá de lo administrativo. El propósito de este estudio es revisar los diferentes modelos de descentralización de la gestión educativa a nivel de gobierno local y determinar qué modelo podría ser apropiado a nuestro contexto con el objetivo de plantear beneficios y riesgos de incluirse en futuras políticas nacionales.

\section{Metodología}

El enfoque del presente estudio es cualitativo, debido a que es un proceso dinámico en el que se hace una revisión de estudios previos en donde las preguntas pueden surgir durante el proceso (Hernández et al., 2014). El tipo de investigación es básica debido a que recolecta datos de la realidad para acrecentar el conocimiento científico como lo indican Sánchez y Reyes (2015); mientras que el diseño de estudio es de revisión sistemática pues se refieren a resúmenes organizados de la recolección de información (Moreno et al., 2018).

La técnica empleada es el análisis documental que implica la recopilación, selección y análisis de datos de diversas fuentes bibliográficas (Pimienta y de la Orden, 2017), además utilizamos el gestor bibliográfico Mendeley que automatizó la recolección de datos y su posterior referenciación. Se seleccionó documentación que cubre tres criterios: el primer criterio es de origen: fuentes nacionales, regionales y globales que hayan tenido experiencia en descentralizar sus gestiones educativas a nivel local; el segundo criterio es definición de conceptos claves como: gestión educativa, descentralización y calidad educativa y finalmente, el tercer criterio es temporalidad: la bibliografía no puede ser mayor a cinco años.

\section{Resultados}

Se presentan los resultados de acuerdo al análisis de cuatro documentos. El primer análisis corresponde al informe titulado Descentralización de la gestión educativa preparado por Luis Chirinos (2011) para Usaid que el acrónimo de US, como Estados Unidos y AID, como ayuda, incorporándose además Del pueblo de los Estados Unidos de América; que describe y proporciona información vigente durante el periodo de descentralización subnacional. Además, el segundo análisis examina el proceso de municipalización y desmunicipalización en Chile; el tercer análisis detalla la experiencia de la gestión educativa municipal en Brasil, y el cuarto y último documento analiza la descentralización como herramienta potencial de cambio en la gestión educativa.

\section{Resultados de la Descentralización de la gestión educativa - Perú}

Luego de revisar la presente publicación podemos concluir que el desarrollo de la municipalización de la gestión educativa en el Perú durante el periodo 2006 al 2011, se presentó dentro de otro proceso que ya había 
iniciado en el año 2002 el cual buscaba descentralizar a todos los sectores del gobierno central. Dicho proceso general tuvo en algunos momentos un apresuramiento y en otros una lentitud en cuanto a la emisión de la normatividad necesaria para consolidarlo. La descentralización de la gestión gubernamental que se planteó a inicios en el siglo XXI encontró inicialmente un consenso de parte de las diferentes tiendas políticas. Se desarrolló un marco legal que permitiría la transición de un gobierno central a uno descentralizado. Para ello se dieron las siguientes leyes: la ley de Bases de la Descentralización, la ley orgánica de Gobiernos Regionales, la ley orgánica de Municipalidades y la ley orgánica del Poder Ejecutivo, siendo esta última promulgada en el ańo 2007 (casi cinco ańos después de las anteriores), entre otras que se emitieron a lo largo del periodo 2002 al 2005. Es a partir de este momento que se paralizó el proceso.

El 28 de julio de 2006, con ocasión de la juramentación del segundo gobierno de Alan García, el presidente anunció un shock descentralista, comprometiéndose a terminar la transferencia de funciones a los gobiernos regionales y lo más saltante en su anuncio fue la creación de un plan piloto de municipalización de la educación y salud, las municipalidades serían responsables directos de la gestión educativa de su distrito o provincia. Es aquí que muchos críticos manifiestan que se estaban dando dos procesos descentralistas paralelos, por un lado, la transferencia de funciones de todos los sectores a las regiones y adicionalmente la municipalización de la educación.

Dicho plan piloto comprendió inicialmente como primera etapa la participación de 56 municipalidades a nivel nacional quedando al final 47. La segunda etapa contemplaba cubrir 110 distritos y la tercera etapa sería a nivel nacional. Los críticos señalan que los gobiernos regionales fueron excluidos de la decisión de municipalizar la gestión educativa y, por lo tanto, las funciones transferidas debían ser compatibilizadas con el nuevo modelo descentralizador. Es evidente que la municipalización no tuvo un desarrollo orgánico que involucrara a todos los actores del proceso descentralista sino una decisión política del poder ejecutivo que en este contexto buscaba el apresuramiento del despliegue de este nuevo modelo.

Se menciona algunos logros que la municipalización de la gestión educativa obtuvo: la consolidación del proceso en 44 municipalidades quienes recibieron viabilidad técnica y 37 fueron acreditadas para la transferencia de funciones. Todas habían establecido su Consejo Educativo Municipal (CEM), entre otros. Se dice que la municipalización estaba llena de incoherencias, por ejemplo, la ley de Bases de Descentralización que señalaba que los gobiernos locales participan en la gestión educativa mas no que son gestores independientes. Se comentó que la duplicidad de estrategias traería problemas y conflictos legales, se resaltó la transferencia de funciones administrativas y de recursos mas no de funciones pedagógicas.

Además, se señala que existe una desigualdad de las capacidades de gestión de las municipalidades lo cual es el reflejo de la heterogeneidad social, económica y cultural. Chirinos (2011) señala que al 2009 no se había aprobado la ley de Organización de funciones del Ministerio de Educación de Perú, el cual debía adaptarse al nuevo modelo de gestión del gobierno, es decir, a la descentralización de este sector y serviría de guía para establecer los lineamientos mismo ministerio, gobiernos regionales y gobiernos locales, la distribución de funciones entre los tres niveles de gobierno (esta Ley se ha aprobado 12 años después, en junio del 2021).

\section{Resultados del Proceso de Municipalización y Desmunicipalización en Chile}

La revisión de esta publicación nos permite concluir que la descentralización educativa es un proceso en constante evolución. Chile ha sido mencionada en diferentes publicaciones como un ejemplo positivo de descentralización subnacional; sin embargo, recientemente ha dado un cambio radical de una educación pública municipalizada a una desmunicipalizada. El libro que es materia de nuestro análisis ilustra en detalle el desarrollo de municipalización de la educación en Chile pasando por sus orígenes como Estado docente a un sistema escolar regido por el mercado. Según Bellei et al. (2018) este modelo de descentralización promovió la privatización convirtiendo a Chile en el país con más privatización educativa en el mundo. Los movimientos estudiantiles del 2006 y del 2011 marcaron un hito que señalaba abiertamente el reclamo de justicia en 
educación. En efecto, el estatus social y de transformación de la educación se había perdido en el mercado educativo acentuando las inequidades sociales.

La transformación educacional en Chile incluyó debates sobre el mercado educativo, así como los problemas de calidad y equidad. El plan de gobierno de Bachelet en el 2014, incluyó una reforma educativa que contempló un cambio de paradigma y reconoció que la educación es un derecho social. En el año 2015, se concreta la llamada Ley de Inclusión que elimina el financiamiento compartido (copago) y prohíbe el lucro de instituciones educativas y regula la admisión estudiantil. Chile ha empezado la desmunicipalización con el reto de construir un nivel intermedio entre el Estado y las instituciones educativas llamado Servicio Local de Educación (SLE). El proceso de desmunicipalización es gradual y se estima que para el año 2030, los SLE que reemplazarán a los municipios llegarán a ser 70 y tendrán como labor hacerse cargo de los más de cinco mil establecimientos educacionales municipales.

Asimismo, en esta publicación se hace un diagnóstico de la educación pública municipal en el que se describe la educación a la fecha de publicación. Chirinos (2011) nos indica que existen cuatro tipos de administradores en el sistema educativo chileno: Municipal (pública), particular subvencionado, particularespagados y Corporaciones de administración delegada. En el año 2017 se observa que el 55\% de la matrícula escolar se encuentra en la administración particular subvencionada, el 36\% en la municipal, el $8 \%$ en la particular pagada, y el $1 \%$ en la administración delegada.

En países de la OCDE (Organización para la Cooperación y el Desarrollo Económico) la participación en educación pública es del $84 \%$ y siendo Chile miembro de esta organización no deja de sorprender que solo llegue a 36\%. Se registra información que desde 1990 ha habido una caída constante en la matrícula escolar en las dependencias municipales. Además, se anota que el sistema ineficiente es el resultado de las pruebas PISA en el que se observa que el sector público obtuvo un rendimiento más bajo que el sector particular subvencionado.

Chirinos (2011) registra información sobre información sobre el rendimiento y asistencia escolar que muestra la dependencia municipal por debajo de las otras dependencias. Ello se manifiesta en la asistencia promedio anual de los alumnos durante el año 2017, que para la gestión municipal es de $90 \%$, para la particular-subvencionada $92 \%$ y para la particular-pagada de $94 \%$. Otro dato de suma importancia es lo referido al informe del Banco Interamericano de Desarrollo (BID, 2018) que manifiesta que el 63\% de locales de gestión municipal poseía un déficit de ingresos para cubrir costos mostrando una mayor deficiencia en establecimientos pequeños y medianos. Es sumamente delicado la revisión del rubro deudas en la que se observa las obligaciones que los municipios han adquirido con respecto mayoritariamente a gastos previsionales adquiridos por 297 municipios alcanzando la suma de 94,000 millones de pesos.

Existen numerosos problemas que afectan la educación pública chilena pudiendo considerarse: la insuficiente gestión municipal, falta de personal debidamente capacitado, así como la desigualdad de recursos debido a que muy pocos municipios pueden financiarse y mantener una saludable posición financiera. Se menciona que la educación pública en el país del sur a alejado a la comunidad debido a la baja calidad de los servicios que brinda y los bajos rendimientos en cuanto a índices educativos.

\section{Resultados de la experiencia de la gestión educativa municipal en Brasil}

El análisis del presente artículo revela el complejo desarrollo de municipalización de la gestión educativa en Brasil y los requerimientos normativos, las políticas públicas desarrolladas con el objetivo de garantizar el derecho al acceso educativo para todos sus pobladores. Según Lagares (2015) el sistema educativo en Brasil es plurisistémico, es decir, los sistemas educativos federales, del distrito federal, estatal y municipal son autónomos entre sí. La Constitución brasilera establece el sistema de distribución de poderes y funciones legislativas entre los componentes del sistema federativo, con marcados límites, aceptando la importancia e independencia de cada uno de ellos y no aceptando incompatibilidades o invasión de competencias. 
En 1987, el Foro para la Defensa de las Escuelas Públicas señalaba que la municipalización de la educación ampliaría la desigualdad entre los gobiernos locales debido a que las municipalidades son heterogéneas en recursos proponiendo que los municipios estén a cargo de la educación infantil y primaria así como que los Estados apoyen directamente a aquellos municipios que tengan mayores dificultades. En los años noventa, las orientaciones neoliberales redujeron la acción estatal y se incrementaron la autonomía de gobiernos subnacionales, favoreciendo la participación ciudadana. El gobierno federal ha dejado las responsabilidades concernientes a los programas sociales en la responsabilidad de los gobiernos subnacionales que son los Estados y municipios.

La heterogeneidad existente en el territorio nacional hace difícil el accionar de los gobiernos municipales. Solo un pequeño porcentaje de municipalidades goza de una posición financiera, política institucional y técnico-administrativa capaz de asumir el rol de gestor autónomo. La descentralización permite escoger las prioridades locales sin tener que consultar al gobierno central. Brasil tiene un enorme territorio e históricas brechas de desigualdad, es un país profundamente heterogéneo por lo que no es acertado esperar el acceso universal de la población al íntegro de programas sociales. Una característica del gobierno brasilero es la gran cantidad de funciones compartidas por las tres estancias de gobierno. Algunos críticos mencionan que la municipalización de la gestión educativa no garantiza la democratización de la educación, sino que a las poblaciones más pobres se le ofrece una educación de baja calidad.

Lagares señala que dentro de las características del presente Estado brasileño se encuentran: las raíces patrimonialistas, relaciones patriarcales, personalistas y clientelistas. Las municipalidades son agentes importantes de la educación brasileña derivando en nuevos enfoques político-educativos por lo que es relevante poner en la mesa de debate las condiciones, metas, debilidades y fortalezas de estos organismos subnacionales. Otro aspecto que se hace necesario analizar es el tema de los sistemas educativos en los municipios, pues la palabra sistema posee diversas interpretaciones y puede ser aplicado de diferentes maneras.

Algunos autores consideran que los sistemas educativos locales constituyen una necesidad debido a la gran complejidad de demandas de la comunidad y como un potencial organismo democratizador. Finalmente, existe un tema permanente por discutir relacionado a cómo construir sistemas educativos a nivel local y un sistema educativo nacional.

\section{Resultados de la descentralización como herramienta potencial de cambio en la gestión educativa.}

Según el documento analizado se puede apreciar que los motivos por los que algunos países se inclinan a descentralizar sus gestiones educativas se orientan en contribuir a una mejora del aprendizaje, desarrollo democrático, desarrollo económico y generar más confianza en el gobierno. Un diseño adecuado del plan de descentralización y la coordinación efectiva de las autoridades puede reducir los efectos negativos que podría generar, como la colisión de políticas, a modo de ejemplo. Esta publicación identifica dos tipos de descentralización: funcional y territorial.

Con relación a la descentralización funcional está se refiere que una autoridad central delega a otras autoridades autónomas competencias, aunque las decisiones son ejecutadas por la autoridad central. Asimismo, la descentralización territorial se lleva a cabo cuando hay una redistribución de competencias entre la autoridad central y autoridades subnacionales, por ejemplo, regiones, distritos, entre otros. A su vez, la descentralización territorial se divide en: desconcentración que es la clase de descentralización en la que la autoridad central crea sucursales bajo su control. La delegación es el proceso de transferir a oficinas locales ejecutoras funciones administrativas, pero no competencias. El traspaso implica la delegación de competencias y responsabilidades a una organización subnacional convirtiéndose esta última en una autoridad autónoma.

Con respecto a la relación directa de la descentralización y a los resultados del aprendizaje, dicho documento manifiesta que hay datos insuficientes acerca de esta relación, aunque se sugiere que la descentralización influye en la cultura del aprendizaje. Los efectos negativos pueden originarse en el potencial riesgo de la generación 
de conflictos entre los diferentes niveles de gobierno debido a una falta de lineamientos; otro efecto negativo podría ser la toma de la gestión educativa por grupos de poder locales. Finalmente, la descentralización está influenciada por los líderes políticos posicionados en el gobierno de turno quienes generalmente son los que impulsan el rumbo de una nación.

\section{Discusión}

El presente artículo buscó comparar los diferentes modelos de descentralización subnacional que se aplicaban en la región y determinar las diferencias existentes entre ellos, analizando el modelo chileno y el modelo brasilero, ambos relativamente vigentes. Encontramos grandes diferencias entre los modelos seńalados líneas arriba. Una de las mayores diferencias entre la experiencia peruana y la chilena es la articulación con el Ministerio de Educación. Mientras en Chile el Estado se apartó totalmente de la gestión de la educación de los centros educativos delegándolos a sus comunas; en el Perú, el Ministerio de Educación no se desvinculó en ningún momento, es más el proceso de descentralización estaba llamado a articular el Ministerio de Educación, los gobiernos regionales y gobiernos locales. Otro aspecto resaltante es que en Chile la municipalización de la educación se desarrolló bajo un modelo educativo de mercado, de fomento a la privatización, competencia y subvención de acuerdo con la demanda; mientras que, el plan piloto en el Perú delegaba funciones administrativas y seguía recibiendo recursos del Estado. Añadimos que Chile debe estar dando por concluida la municipalización de la educación pública para el año 2030.

El análisis de la experiencia brasilera en comparación con la experiencia peruana nos da como resultado marcadas diferencias en lo que respecta a dimensiones de tiempo y organización política. Brasil lleva cerca de 40 años aplicando la municipalización educativa en inicial y primaria y Perú empezó el plan piloto en el año 2006 y lo dio por concluido en el año 2011. Otra gran diferencia es que Brasil es un país plurisistémico donde se da la descentralización, unificación al mismo tiempo y como país federal existe cierta autonomía entre los gobiernos nacionales, estatales y municipales. No existe un sistema educativo organizado a nivel nacional sino diversos subsistemas; por su lado, el Perú es un país unitario y descentralizado, según la Constitución Política vigente y existe un solo sistema educativo bajo la rectoría del Ministerio de Educación.

El análisis del Instituto Internacional de Planeamiento de la Educación de la Unesco propone que una descentralización educativa bien diseñada con acompañamiento y revisiones periódicas puede ser efectivo para promover el desarrollo educativo, cultural y democrático de una nación; además de brindar la posibilidad de alcanzar un mayor grado de eficiencia en la administración de recursos fiscales. La descentralización subnacional es generalmente bienvenida en países que tienen una tradición de gobiernos autónomos.

\section{Conclusión}

Municipalizar el sector educación, es un proceso sujeto a constantes cambios, necesita de diagnósticos periódicos para evaluar el logro de sus objetivos, grado de eficiencia y alcance. Los modelos de municipalización de la educación son diversos y es necesario reconocer que un resultado favorable o desfavorable desde estas organizaciones locales parte de la voluntad política y el rumbo que marquen los gobiernos centrales. En el caso peruano los resultados obtenidos nos llevan a reflexionar sobre la importancia de diseñar y planificar en detalle los instrumentos y herramientas en conjunto con todos los actores involucrados, el shock descentralizador del 2006 estuvo caracterizado por una aceleración del proceso descentralista que fue criticado por considerarse que duplicaba funciones entre los gobiernos subnacionales.

América Latina es reconocida como una región heterogénea en cuanto a geografía, desarrollo económico y social. Delegar competencias a sus municipios posee como riesgo marcar más aun una profunda desigualdad en cuanto a la justicia educativa; sin embargo, posee beneficios. En el caso de Brasil, la municipalización determina prioridades sin que haya que esperar que el gobierno nacional apruebe sus decisiones. Ello debido a que Brasil posee un gobierno federal, es decir, existe una autonomía federal, estatal y municipal, distinto al peruano. 
El fracaso de un modelo como el chileno no determina que los otros estén condenados a extinguirse, muy por el contrario, nos invita a reflexionar sobre la experiencia de este en otras naciones. Esto se realiza con la finalidad de fortalecer el potencial de desarrollar un modelo propio que contemple un proceso de evaluación periódica que incluya sistemas de información y capacitación permanente para docentes, directivos y personal administrativo y sobre todo que se identifique quienes necesitan un acompañamiento permanente y mayores recursos fiscales.

\section{Referencias}

Banco Mundial (2018). World Development Report 2018 [Informe sobre el desarrollo mundial 2018]. Banco Mundial digital library.

Bellei, C.; Muñoz, G., Rubio, X.; Alcaíno, M.., Donoso, M. Martínez, J., de la Puente, L.; del Pozo, F., y Díaz, R. (20118). Nueva Educación Pública. Contexto, contenidos y perspectivas de la desmunicipalización. Universidad de Chile. https://www.educacionpublica.cl/wp-content/uploads/2018/11/DEP.pdf

Banco Interamericano de Desarrollo (2019). Del papel a la nube. Cómo guiar la transformación digital de los Sistemas de Información y Gestión Educativa (SIGED). BID digital library. http://dx.doi.org/10.18235/0001749

Canazas, K., y Márquez, K. (2015). Diagnóstico del programa de municipalización de la gestión educativa en el marco de la descentralización: estudio de caso de la municipalidad de Ventanilla [Tesis de maestría, Pontificia Universidad Católica del Perú]. https://bit.ly/3zlLkAj

Cerro, C. (2020) El nuevo Sistema de Educación Pública Chileno: Fundamentos, elementos de su puesta en marcha y situación actual [Tesis de maestría, Universidad de Talca]. https://bit.ly/3zqsKqD

Chacón, L. (2014). Gestión educativa del siglo XXI: bajo el paradigma emergente de la complejidad. Ommia, 20(2). https://bit.ly/3iDc9Jo

Chirinos, L. (2011) Descentralización de la gestión educativa. USAID PERU SUMA. https://pdf.usaid.gov/pdf_docs/ PA00JXTZ.pdf

Hernández, R., Fernández, C., y Baptista, M. (2014). Metodología de la investigación (6a ed.). Mc Graw Hill Education.

Hernández, R., y Mendoza, C. (2018). Metodología de la investigación. Las rutas cuantitativa, cualitativa y mixta. McGraw-Hill Interamericana. https://bit.ly/3zsatcN

Instituto Internacional de Planificación Educativa-Organización de las Naciones Unidas para la Educación, la Ciencia y la Cultura. (2021). Descentralización de la gestión de las escuelas. https://bit.ly/3kOdwbf

Instituto Internacional de Planificación Educativa-Organización de las Naciones Unidas para la Educación, la Ciancia y la Cultura (2018). 3bis. Decentralization: pros and cons. https://bit.ly/3eEN3IX

Jiménez, J. (2019). Transformando la educación desde la gestión educativa: hacia un cambio de mentalidad. Praxis, 15(2), 223-235. https://bit.ly/3BrzLtk

Kameshwara, K., Sandoval, A. Shields. R., \& Rai, K. (2020). A false promise? Decentralization in education systems across the globe [Una falsa promesa? Descentralización en los sistemas educativos de todo el mundo]. International Journal of Educational Research, 104. https://doi.org/10.1016/j.ijer.2020.101669

Lagares, R. (2015). Institucionalização de sistemas municipais de educaçấo: concepçôes e complexidade [Institucionalización de los sistemas educativos municipales: conceptos y complejidad]. Roteiro, 40(1), 127-148. https://doi.org/10.18593/r.v40i1.4268

Ministerio de Educación de Perú (2015) Lineamientos para la Gestión Educativa Descentralizada. Resolución de Secretaría General No 938. https://bit.ly/3rqP2pC

Moreno, J. (2021). Descentralización educativa en el municipio certificado de Tunja: un proceso complejo en tiempo de postconflicto. Centros Año 10, (1). 68-81. https://doi.org/10.48204/j.centros.v10n1a5 
Moreno, B., Muñoz, M., Cuellar, J., Domancic, S., y Villanueva, J. (2018). Revisiones Sistemáticas: definición y nociones básicas. Revista clínica de periodoncia, implantología y rehabilitación oral, 11(3), 184-186. https://scielo. conicyt.cl/pdf/piro/v11n3/0719-0107-piro-11-03-184.pdf

Organización de las Naciones Unidas para la Educación, la Ciencia y la Cultura (2016). Educación 2030. Declaración de Incheon y Marco de acción hacia una educación inclusiva y equitativa de calidad y un aprendizaje a lo largo de la vida para todos. UNESCO Digital Library. https://bit.ly/3BrAdI2

Organización de las Naciones Unidas para la Educación, la Ciencia y la Cultura (2020). Informe de seguimiento de la Educación en el Mundo, 2020, América Latina y El Caribe: Inclusión y Educación: Todos y Todas Sin Excepción. UNESCO Digital Library. https://bit.ly/3xWkluN

Organización para la Cooperación y el Desarrollo Económicos (2018). How decentralised are education systems, and what does it mean for schools?. Education indicators in focus, November 2018, number 64 [¿Qué tan descentralizados están los sistemas educativos y qué significa para las escuelas? Indicadores de educación en foco, noviembre de 2018, número 64]. https://bit.ly/3izsOxB

Pimienta, J., y de la Orden A. (2017). Metodología de la investigación. (3a ed.). Pearson Educación de México S.A. de C.V.

Ríos, A. (2020). Gobernabilidad y gobernanza en las Unidades de Gestión Educativa Local del Perú: una mirada desde el conocimiento de sus funciones. Revista Iberoamericana de Educación, 83(1), 97-119. https://doi.org/10.35362/ rie8313832

Ríos, J. (2017). Factores que influyeron en la implementación del Programa de Municipalización de la Educación en el distrito de San Juan Bautista de Loreto durante los años 2007 - 2011 [Tesis de maestría, Pontificia Universidad Católica del Perú]. http://hdl.handle.net/20.500.12404/12822

Rivas, A. (2015). América Latina después de PISA: Lecciones aprendidas de la educación en siete paises (2000 - 2015). Fundación CIPPEC. https://bit.ly/3BpVlON

Sánchez, H., y Reyes, C. (2015). Metodología y diseños en la investigación (5ª ed.), Aneth SRL Lima. 